\title{
Detection of network anomaly based on hybrid intelligence techniques
}

Shahbaa I. Khaleel Karam mohammed mahdi saleh

shahbaaibrkh@uomosul.edu.iq

College of Computer Sciences and Mathematics

University of Mosul

\section{Received on: 11/09/2011}

\section{Accepted on:14/12/2011}

Artificial Intelligence could make the use of Intrusion Detection Systems a lot easier than it is today. As always, the hardest thing with learning Artificial Intelligence systems is to make them learn the right things. This research focuses on finding out how to make an Intrusion Detection Systems environment learn the preferences and work practices of a security officer, In this research hybrid intelligence system is designed and developed for network intrusion detection, where the research was presented four methods for network anomaly detection using clustering technology and dependence on artificial intelligence techniques, which include a Genetic Algorithm (GA) and Particle Swarm Optimization (PSO) to develop and improve the performance of intrusion detection system. The first method implemented by applying traditional clustering algorithm of KM in a way Kmeans on KDDcup99 data to detect attacks, in the way the second hybrid clustering algorithm HCA method was used where the Kmeans been hybridized with GA. In the third method PSO has been used. Depending on the third method the fourth method Modified PSO (MPSO) has been developed, This was the best method among the four methods used in this research.

Keywords: Artificial Intelligence, Intrusion Detection Systems, Swarm Optimization, Genetic Algorithm, clustering algorithm.

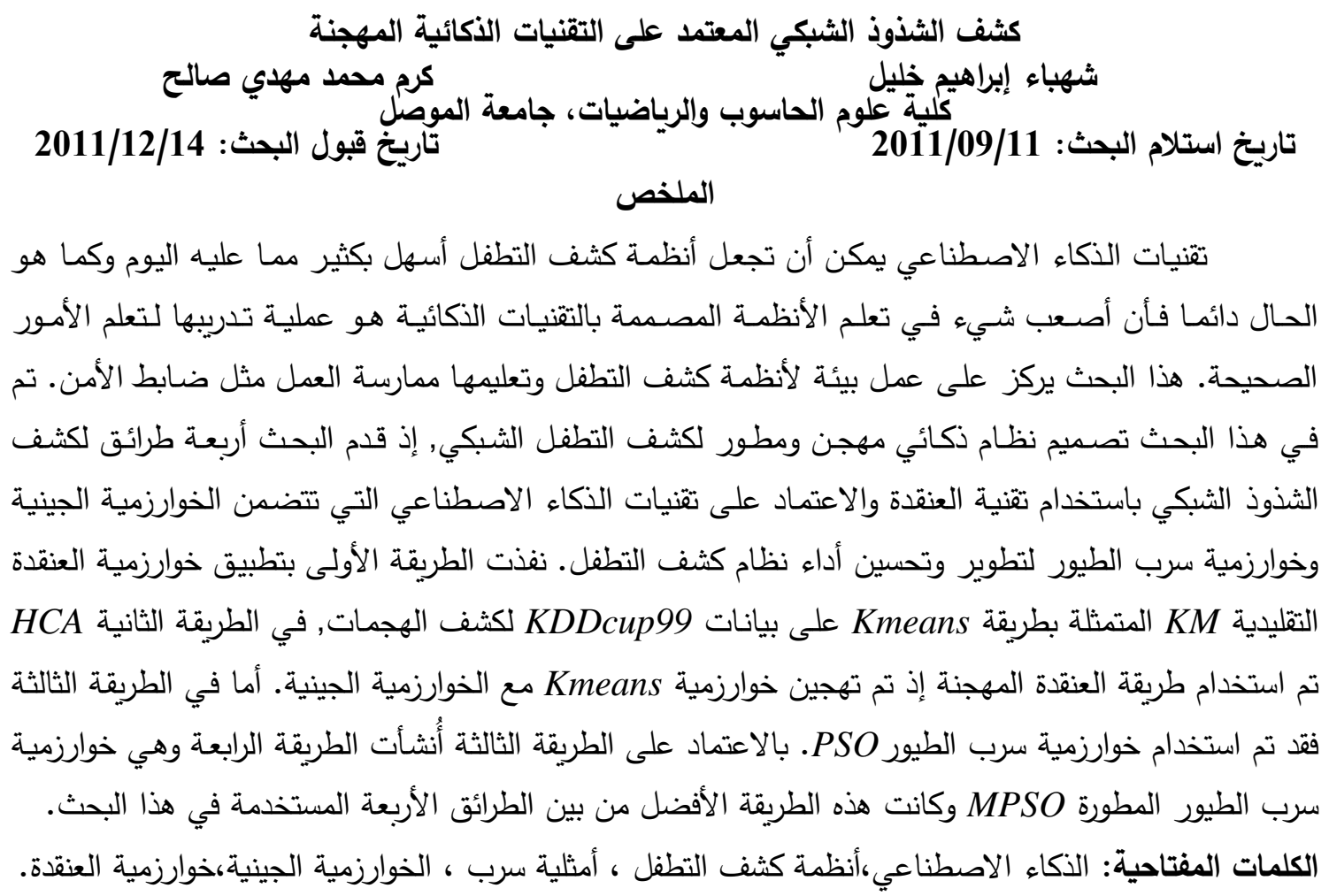


في السنوات الأخيرة، أصبحت أنظمة كثف التطفل IDS واحدة من المناطق الأكثر سخونة في البحوث الخاصـة بأمن الحاسوب. وهي تكنولوجيا كثف مهمة، تستخدم بوصفها إجراء مضاداً للحفاظ على سلامة البيانات واستمرارية

عمل النظام خلال عملية الاقتحام [1]. كثف التطفل يتيح رصد وتحليل نشاط المستخدم والنظام، تدقيق تكوينات النظام ونقاط الضعف، تقييم سلامة النظام وملفات البيانات، التحليل الإحصائي لنشاط النماذج patterns على أساس المطابقة لهجمات معروفة، تحليل

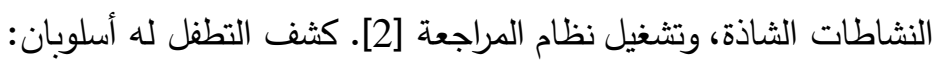

الكثف عن الثذوذ: يثير إلى التقنيات التي تحدد وتميز السلوك العادي أو المقبول للنظام على سبيل المثال،

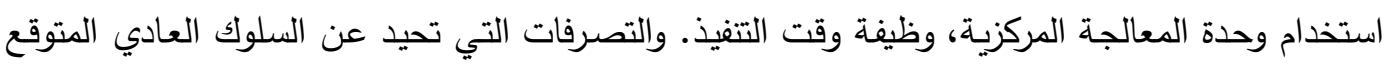
تعدها اختراقات.

الكثف عن إساءة الاستخدام: يشير إلى التقنيات التي تميز طرائق معروفة لاختراق النظام. تتميز هذه الاختراقات بأنها نماذج patterns أو توقيع signature مخزونة في قاعدة المعرفة للنظام لذلك يقوم نظام كثف التطفل بالبحث عن النماذج والتواقيع المشابهة لها ويعدها اختراقات. النماذج أو التواقيع قد تكون فئاعن

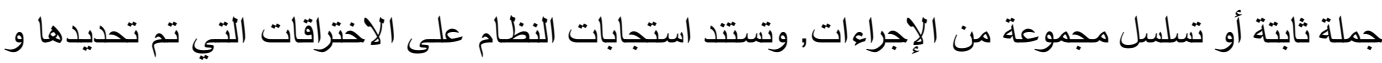

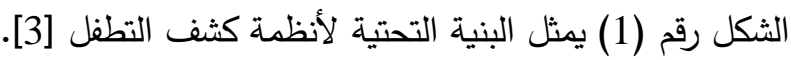

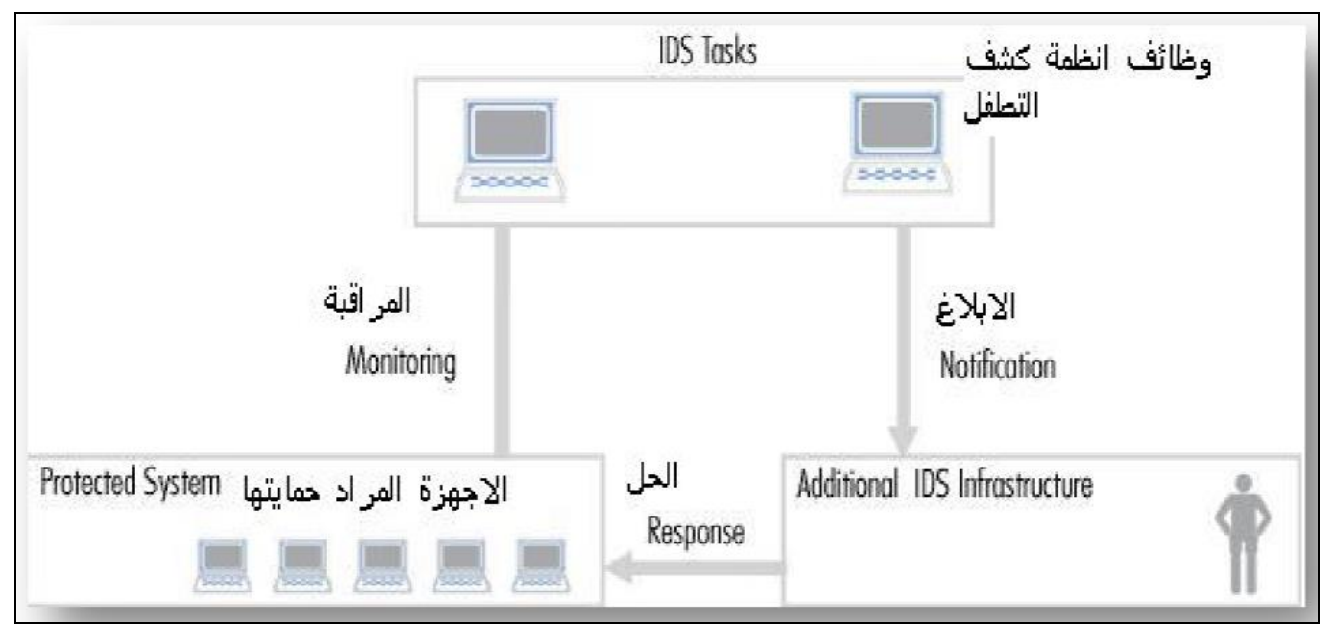

الثكل رقم (1). البنية التحتية لأنظمة كثف التطفل

نظام كشف التطفل من ناحية مجال العمل والحماية يقسم بصورة عامه إلى قسمين:

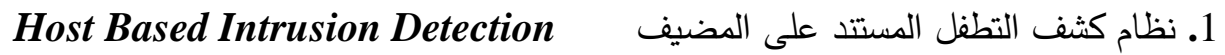
ويرمز له HIDS يقوم هذا النوع من الأنظمة بتحليل الأحداث الموجودة في جهاز الحاسوب ويقوم بتمييز الأحداث والفعاليات الخاصة بالمستخدمين الذين يقومون بنشاطات عدائية ومضرة بنظام التثغيل. يقوم النظام

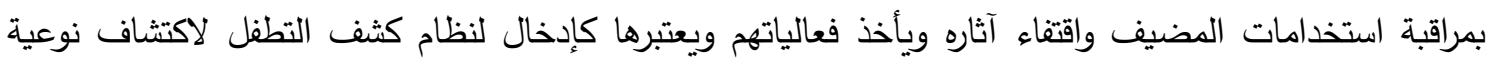

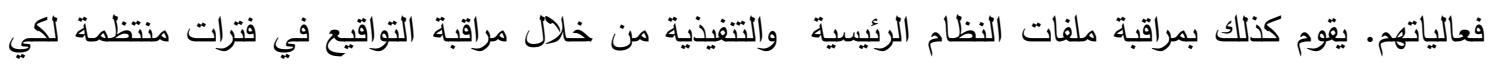

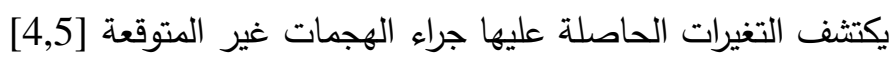


2. نظام كثف التطفل المستتد على الثبكة Network Based Intrusion Detection ويرمز لله NIDS، لا يقوم هذا النوع من أنظمة كثف التطفل باختبار وفحص سجلات تدقيق الأثر الخاصة بالمضيف ، ولكن يقوم بعمل تسجيل وتحليل لفعاليات الشبكة بتحليل البيانات الموجودة في حزم الشبكة من مختلف أجزاء الشبكة ثم يقوم بإصدار تقارير إلى وحدة الإدارة المركزية للنظام عن حالة سريان البيانات المراقبة

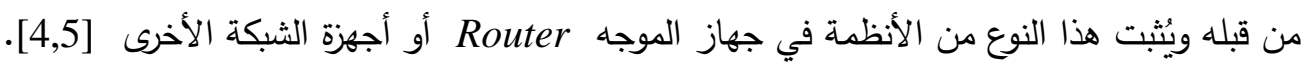

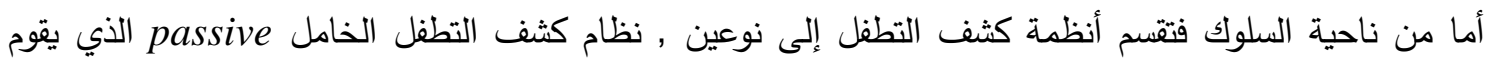

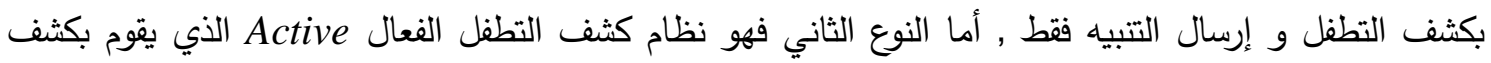
التطفل وأيضا استخدام تقنيات خاصة لمواجهتها وحماية النظام [4,5]. ومن ناحية تحليل البيانات تقسم أنظمة كثف التطفل إلى نوعين النوع الأول يكون نظام معتمد على الزمن الحقيقي حيث يتم مراقبة البيانات وتحليلها

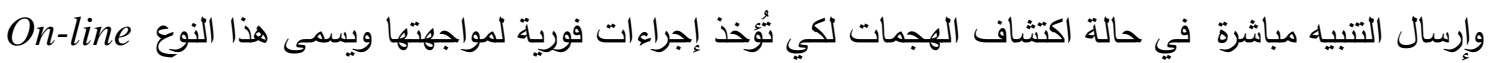
analysis

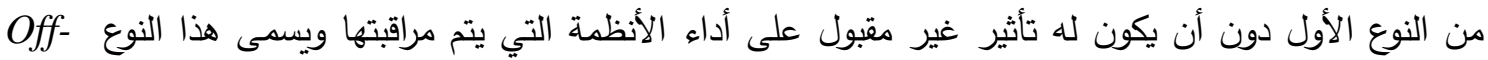
.[4] line analysis 1.1 (الداسات السابقة لقد تم دراسة مشكلة كثف التطفل وتصنيفه ضمن حقل أمنية شبكة الحاسوب ففي عام 2002. قدم

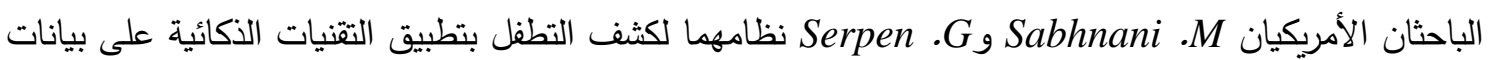
الـ KDD99 لكثف التطفل بصيغة إساءة الاستخدام. عمد الباحثان على تطبيق أنظتهم وبمختلف التقنيات الذكائية على هذه البيانات وكانت تقنياتهم ت تضمن عدة حقول مثل الثبكات العصبية الاصطناعية والمنطق المضبب والنماذج الإحصائية والنماذج الاحتمالية وأثجار القرار، وقد تم تطبيق تسعة خوارزميات من مختلف الحقول المذكورة وبناء نموذج متعدد التصنيف. وقد جاءت نتائج التطبيق متفاوتة بين الخوارزميات مثلا نتائج

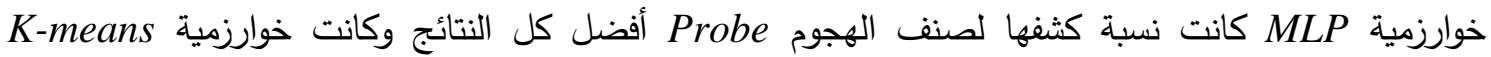
الأفضل بنتائج الكشف الخاصة بصنفي الهجوم DOS و U2R وخوارزمية Gaussian كانت نسب كثفها لصنف الهجوم R2L الأفضل. وقد تم الاستنتاج بأن لكل خوارزمية القدرة على كثف صنف معين من أصناف الهجوم أكثر من غيرها. في عام 2005 استخدم الباحثون .Gary S. Bing C., Annie S. , Kien A الخوارزمية الجينية لاختيار مجموعة جزئية من الخصائص الداخلة إلى المصنف الذي بني باستخدام خوارزمية أثجار القرار C4.5 لزيادة نسبة كثف التطفل وتقليل نسبة الإنذارات الكاذبة وقد تم استخدام مجموعة بيانات KDD99 في

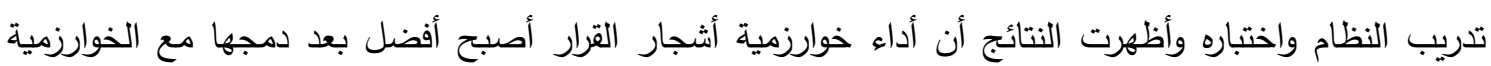

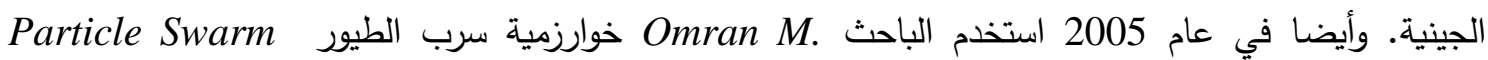
المعتمدة على خوارزميات العنقدة التقليدية في تصنيف الصور بدون معلم وأظهرت النتائج

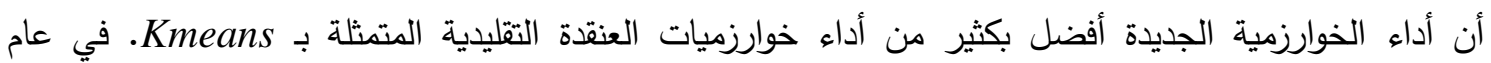
2011 أقترح الباحث .Liu y أسلوباً جديداً لكشف الثذوذ الثبكي عن طريق ربط شبكة دالة القاعدة الثعاعية Particle Swarm Optimization PSO مع خوارزمية سرب الطيور Radial Basis Function RBF 
وقد تم استخدام مجموعة بيانات KDD99 في النظام وأظهرت النتائج أن أداء شبكة دالة القاعدة الثعاعية أصبح

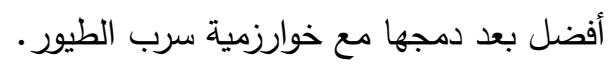

\section{2. وصف المتطلبات Requirements specification}

تلعب المتطلبات دوراً أساسياً خلال عملية إنثـاء المنتج. يتم توثيق المتطلبات في وثيقة تسمى وثيقة وصف المتطلبـات. استخراج المتطلبـات Requirements هو نشـاط مهم في عمليـة هندسـة البرمجيات يهدف إلى اكتساب وفهم المتطلبات. الثكل رقم(2) يمثل مخطط استخراج متطلبات النظام.

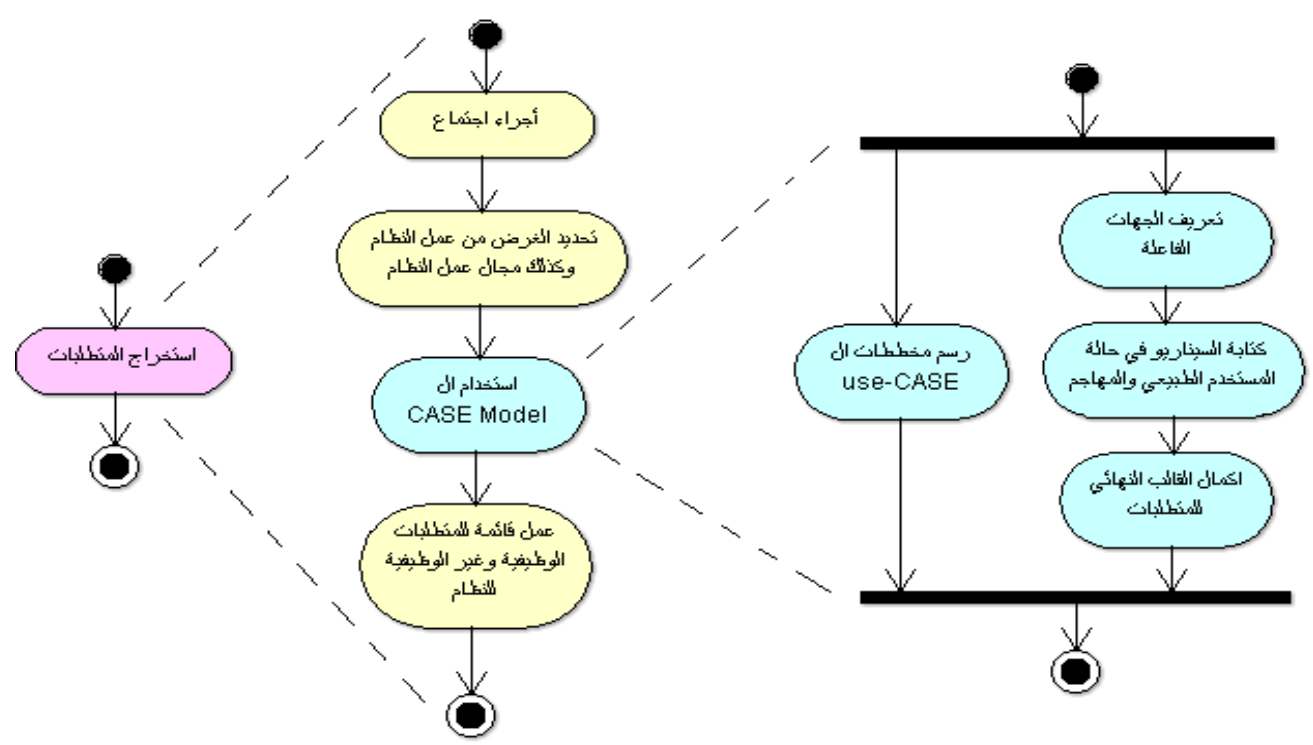

الثكل رقم (2). مخطط UML activity لاستخراج الدتطلبات

Problem 1.2 المشكلة P

نظم وشبكات المعومات تخضع دائماً للهجمات الإلكترونية, ومحاولات اختراق أمن المعلومات تتزايد كل

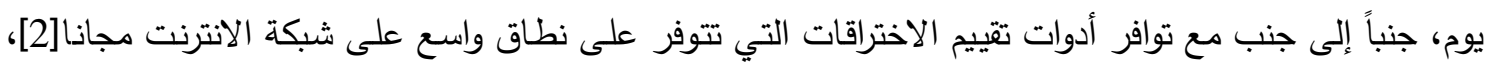

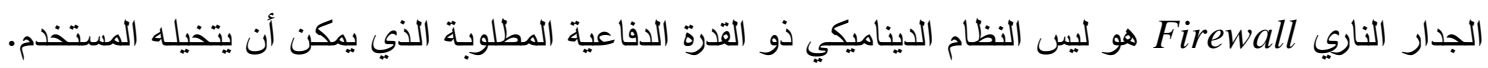

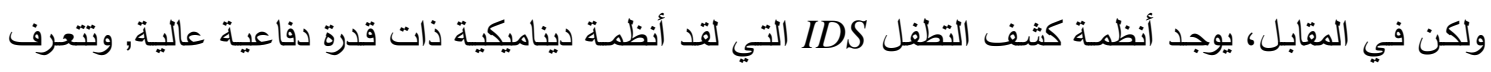
أنظمة كثف التطفل على الهجمات التي لا تستطيع الجدران النارية التعرف عليها [6].

Purpose 2.2

سيقوم النظام باستخدام تقنيات الذكاء الاصطناعي لتحليل البيانات واكتثاف المتطفل إذا حاول الهجوم, إذ إذ إذاء يقوم النظام بتوليد تتبيه يتم عن طريقه إخطار مسؤول النظام لاتخاذ إجراءات معينة إذا لزم الأمر.

Scope 3.2

إن مجال عمل أنظمة كثف التطفل تثمل أنواع وكميات كبيره من النظم التي يتعين دعمها، إذ يمكن أن

يكون النظام المحمي آلة فردية أو شبكة من الأجهزة [7]. 
4.2 استخدام مخطط هندسة البرمجيات بمساعدة الحاسوب Use-CASE Model

تستخدم هذه المخططات في هندسة البرمجيات لاستخراج المتطلبات الوظيفية للنظام. وتقوم أيضاً بتعريف التفاعل بين المستخدمين Actors والنظام [8]. تم استخدام ArgoUML لتطبيق الـAse-CASE على النظام المصمم في هذا البحث. ArgoUML هي أداة تصميم قوية سهلة الاستخدام، تدعم تصميم البرمجيات الرسومية وتطوير وتوثيق تطبيقات البرمجيات[9]، يوجد ثلاث جهات فاعلة Actors أساسية في النظام IDS وهم مسؤول

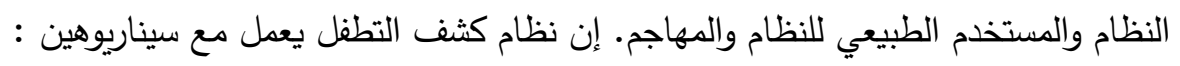

1.4.2 مستخدم طبيعي يستخدم النظام

خـلال الاستخدام العـادي للنظـام، سوف يقوم النظام برصد كل حركات المرور في الجهاز أو الثبكة المحمية. في حالة من النشاط العادي يجب أن يبقى النظام هادئاً ولا يرد على أي شيء طبيعي، ويوفر الخدمات

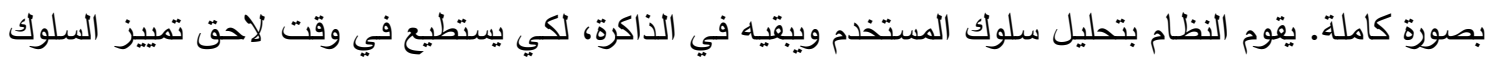
الثاذ كما في الثكل(3). الجهات الفاعلة: المستخدم الطبيعي للنظام.

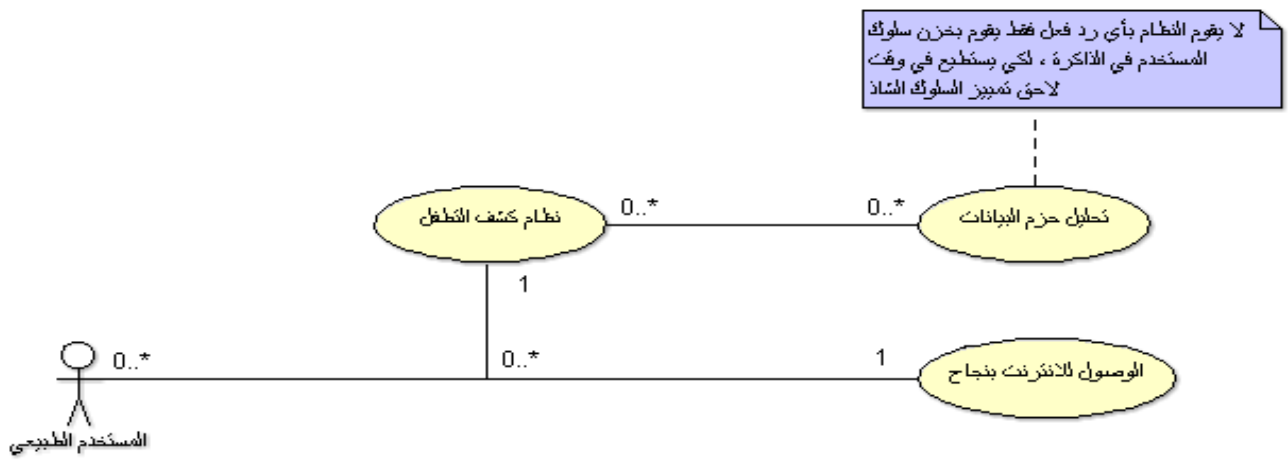

الشكل رقم (3). مخطط UML Use case للمستخدم الطبيعي للنظام

2.4.2 شخص يحاول مهاجمة النظام

في مرحلـة معينـة نظـام كثف التطفل يميز هجمـة معينـة أو إسـاءة استخدام، لذلك يقوم بتوليد إنذار

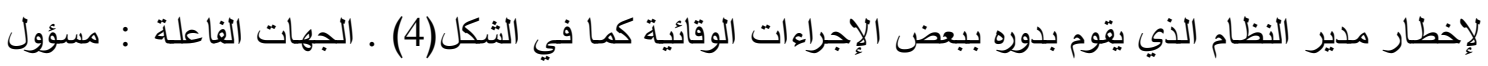

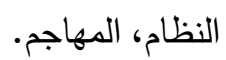

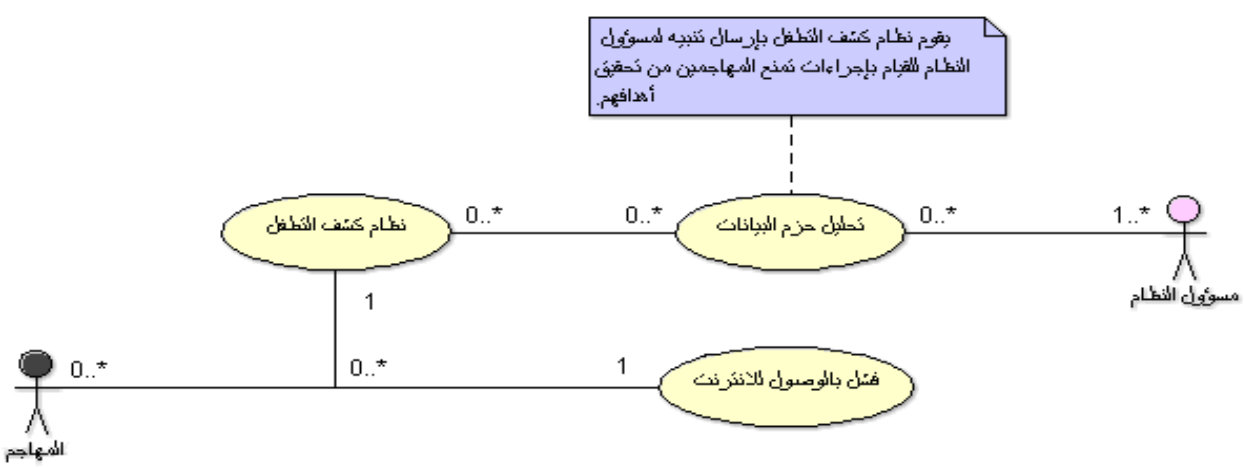

الثكل رقم (4). مخطط UML Use case لثخص يحاول مهاجمة النظام 


\section{Functional Requirements 5.2}

• يجب أن يكون النظام قادراً على قراءة الحزم Packets من مجموعة متتوعة من الدصادر. • • على الدستخدم أن يكون قادراً على تدريب النظام.

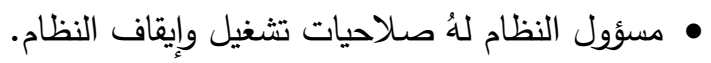

• • النظام يكون قادراً على تعلم الفرق بين الحزمة الطبيعية وغير الطبيعية وتصنيف الهجمات لأنواعها.

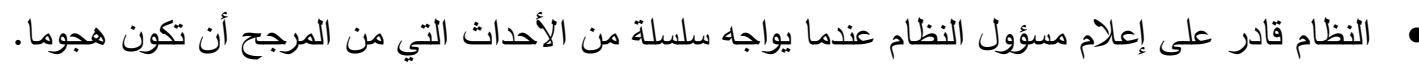
• • • •مع الإحصاءات من الحزم وخزنها في قاعدة المعرفة ليكون النظام قادراً على التحليل والمراجعة.

Non-Functional Requirements المتطلبات الغير وظيفية 6.2 • يجب أن يكون النظام سهلاً لتندريب المستخدمين الجدد على هذا البرنامج. • • على المستخدم أن يكون قادرا على تثغيل وإيقاف النظام بسهولة. • • ضبط معلمات النظام والتحكم فيها بسهولة. • الموثوقية : Reliability : مسؤولو النظام لا يحتاجون لإعادة بدء تثغيل النظام في أي لحظة إلا لأسباب الصيانة الضرورية.

• الأداء Performance : يجب أن يتم التقاط الحزم بسرعة كافية لتمكين تحليل البيانات خلال وقت قصير،

$$
\text { ومن ثُّ إخطار مسؤول النظام في أقرب وقت مدكن. }
$$

• التعزيز Supportability : التكوين الحيوي للنظام يسمح لمسؤولي النظام بإضـافة وتغيير الأوزان والمعلمات النسات دون التأثير على خدمات النظام، ودون تعطيل أي من الخدمات الأخرى على شبكة الاتصال.

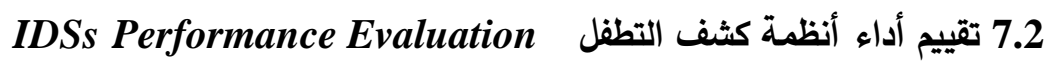

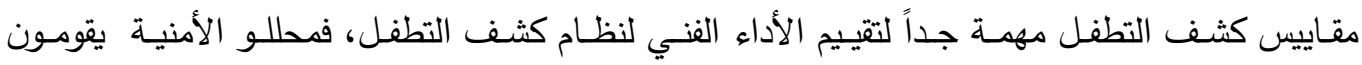
بالإطلاع على مخرجات أنظمة كثف التطفل لكي يتعرفوا على إمكانية حدوث الهجوم ومتى يتم إصدار الإندان الإنارات.

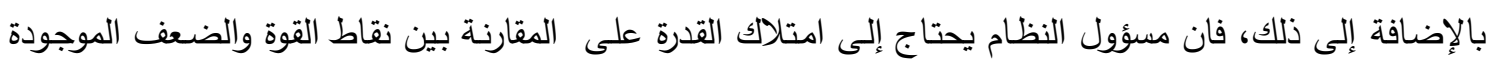

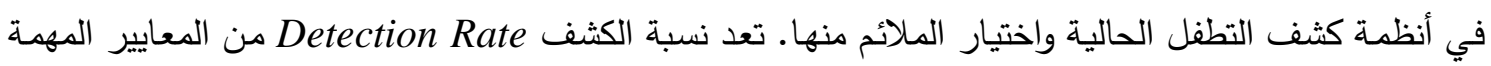
لقياس أداء تُظم كثف التطفل ويتم قياسها حسب المعادلة التالية[10]: $D R=\frac{T P}{T P+T N} * 100$

حيث الـTP هو معيار لقياس عدد سجلات الهجوم التي يتم تصنيفها بصورة صحيحة، وTN هو التها معيار لقياس

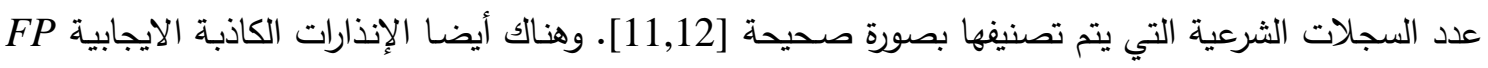

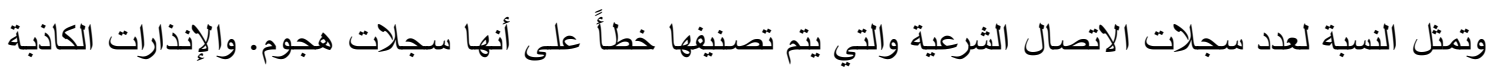

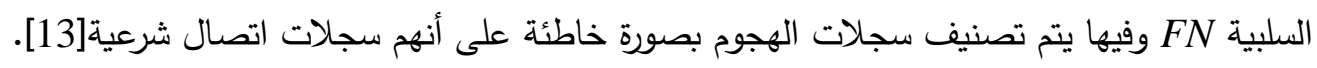


3. تحليل و تصميم النظام Analysis and design of the system

تضمن هذا البحث محورين أساسيين من محاور الذكاء الاصطناعي لكثف التطفل المِحور الأول يتمثل بالخوارزمية الجينية Genetic Algorithm والمِحور الثاني يتمثل بخوارزمية سرب الطيور optimization خوارزمية سرب الطيور المطورة modified particle swarm optimization التي استخدمت للغرض نفسه ولكن بنتائج أفضل وفيما يلي الثكل رقم(5) يمثل مخطط تحليل سير البيانات في نظام كثف التطفل الذي أُعتمِدَ في هذا البحث.

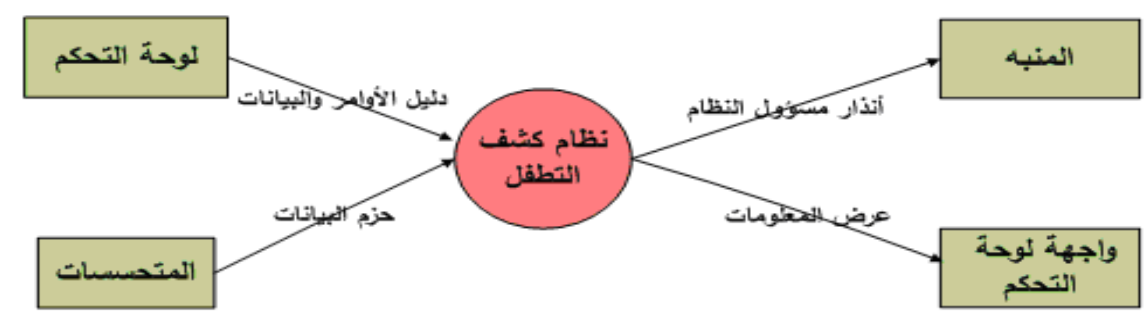

الثكل رقم (5). يمثل المرحلة رقم صفر لرخطط سير البيانات في النظام

ثم يتم تحليل نظام كثف التطفل إلى وحدتين وحدة خاصة بمراقبة المتحسسات ووحدة التحليل كما في

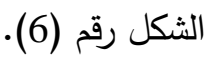

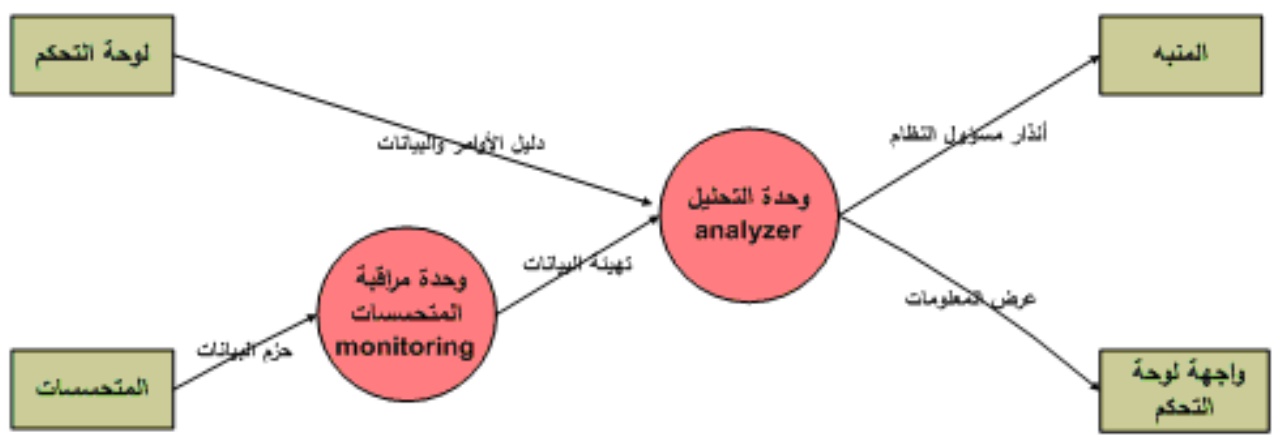

الثكل رقم (6). يمثل المرحلة رقم 1 لمخطط سير البيانات في النظام

وحدة التحليل تمثل قلب النظام حيث تقسم إلى وحدة التدريب التي تحتوي على أسلوب كثف التطفل المعتمد في هذا البحث مثلا الخوارزمية الجينية أو خوارزمية سرب الطيور إذ يتم تدريب وتعليم النظام على التمييز

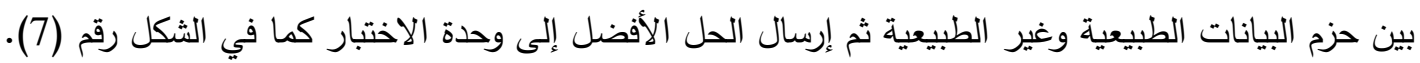




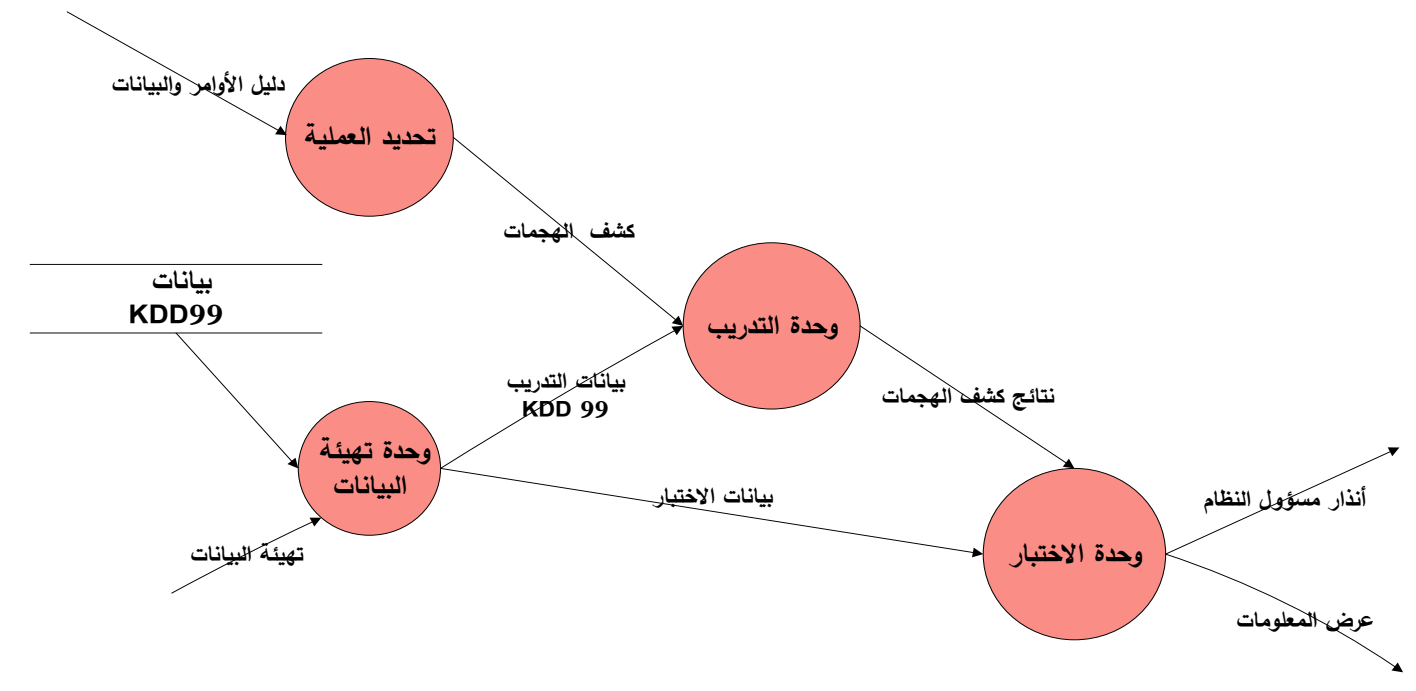

Analyzer الثكل رقم (7). يمثل المرحلة رقم 2 لمخطط سير البيانات في وحدة التحليل

أما البيانات المستخدمة في عملية التدريب والاختبار هي مجموعة بيانات KDD99 وهي لقد البيانات الأكثر استخداماً في بناء نظم كثف التطفل منذ عام 1999 [14]. ولكن قبل استخدام هذه البيانات يتم إدخالها على وحدة ما قبل معالجة البيانات وهي وحدة تهيئة البيانات الخاصة بالنظام التي تقسم بدورها إلى وحدة التحويل Transformation إلى الثكل الرقمي وكذلك وحدة التطبيع للبيانات normalization التي تقوم بتحسين دقة وكفاءة الخوارزميات المستخدمة بحيث يصبح المدى المستخدم للبيانات هو [ 1.0 - 0.0 ] [15]. كما موضح في الثكل رقم (8).

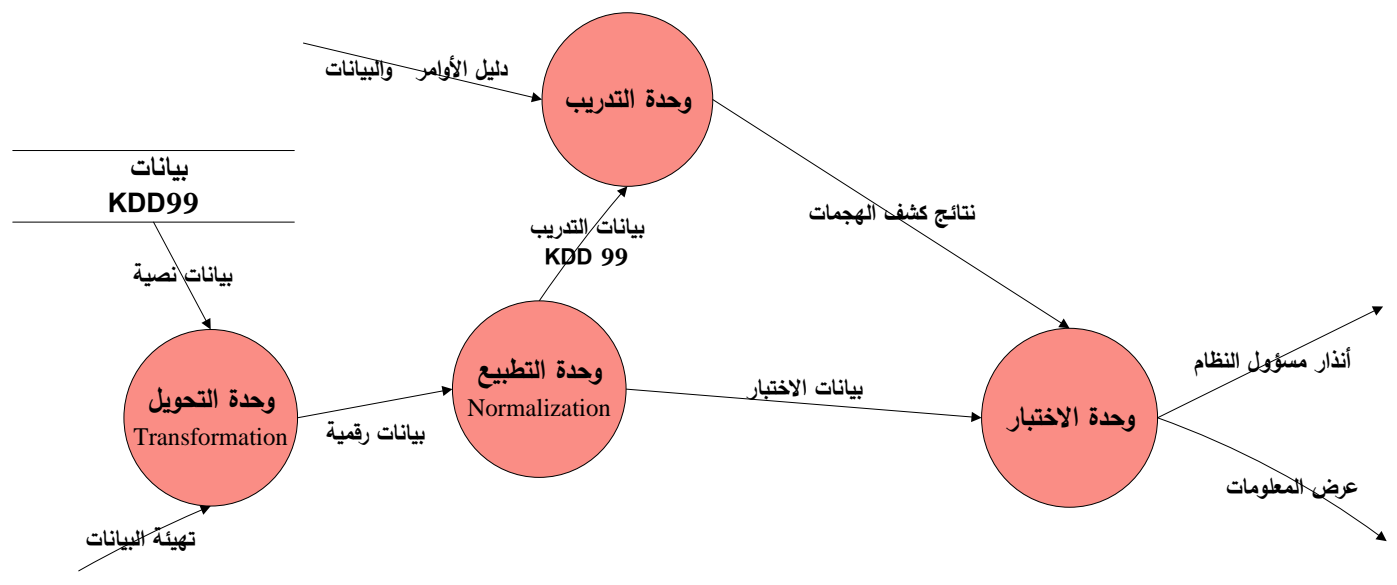

الثكل رقم (8). يمثل المرحلة رقم 2 لمخطط سير البيانات في وحدة تهيئة البيانات

1.3 عنقدة البيانات Data Clustering

العنقدة هي عملية تقسيم البيانات إلى مجاميع اعتمادا على بعض المقاييس المتثابهة لهذه المجاميع ولقد

عملية عنقدة البيانات عملية أساسية ومركزية في الذكاء الاصطناعي إذ يتم تعريف العنقود بواسطة مركز العنقود cluster center Euclidean distance 


$$
d\left(Z_{u}, Z_{w}\right)=\sqrt{\sum_{j=1}^{N d}\left(z_{u, j}-z_{w, j}\right)^{2}}=\left\|Z_{u}-Z_{w}\right\|_{2}
$$

خوارزمية العنقدة الأكثر شيوعا هي خوارزمية K-means التكرارية حيث تقوم هذه الخوارزمية بتقليل المسافة بين

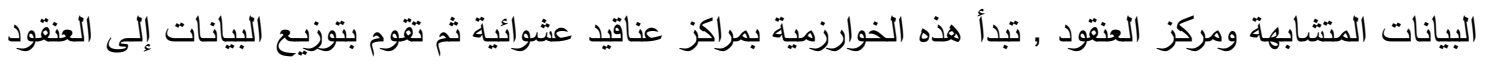

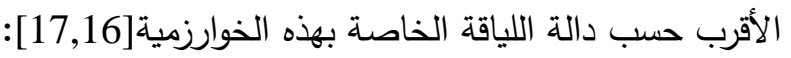

$$
J_{K \text {-means }}=\sum_{k=1}^{K} \sum_{\forall z_{p} \in C_{k}} d^{2}\left(z_{p}, m_{k}\right)
$$

\section{3 الخوارزمية الجينية Genetic Algorithm}

الخوارزمية الجينية لقد من النماذج الحسابية القائمة على مبادئ التطور والانتقاء الطبيعي . هذه

الخوارزميات تقوم بنمذجة المشكلة في مجال معين باستخدام الكروموسومات وتطور هذه الكروموسومات باستخدام الاختيار selection، وإعادة التركيب recombination، وعملية الطفرة mutation. في تطبيقات أمن الكمبيوتر

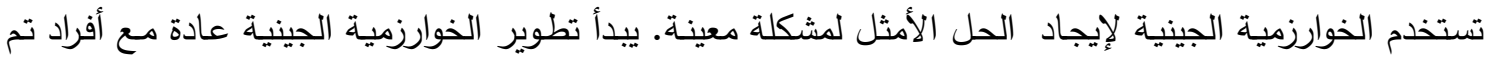

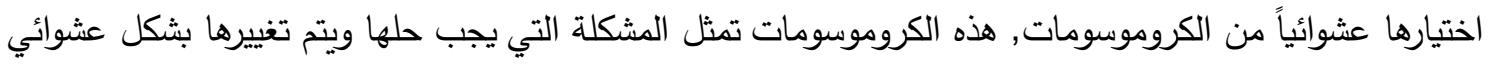
خلال التطور , يطلق على مجموعة من الكروموسومات أثناء مرحلة التطوير مجتمع population. يتت استخدام

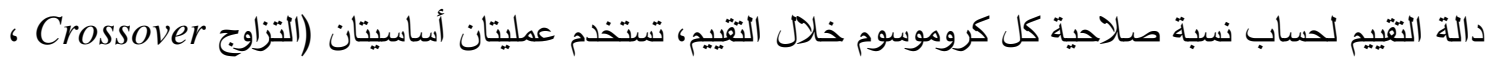

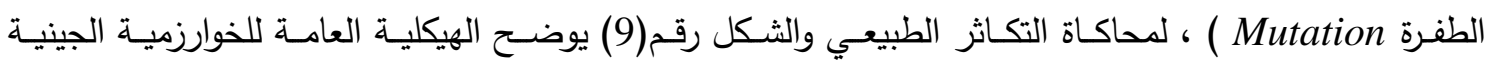

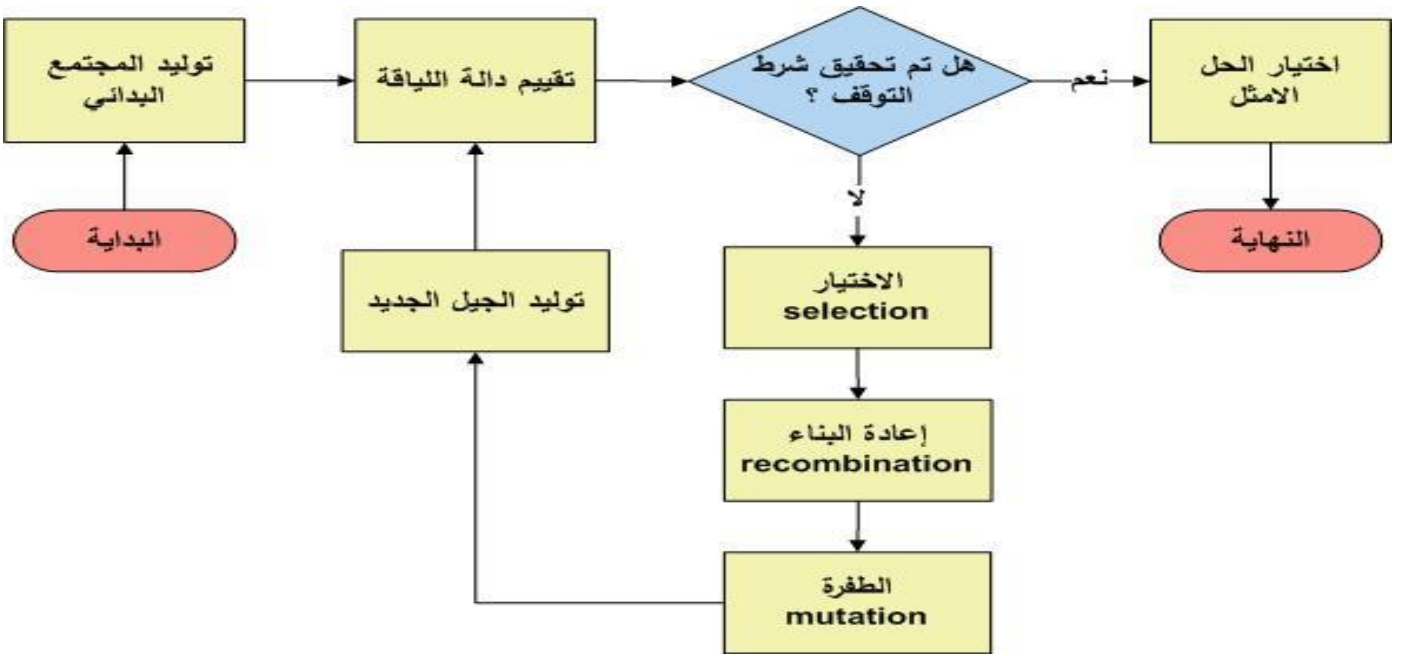

الثكل رقم (9). يمثل الهيكلية العامة للخوارزمية الجينية

أثبتت الدراسات أن هناك مجالاً واعداً في استخدام الخوارزمية الجينية لكثف التطفل. حيث تستخدم لتقييم حزم البيانات التي تمر بالشبكة وتمييز الحزم الطبيعية عن الثاذة التي تدل على احتمالية حدوث اختراق[1]. 


\section{3 خوارزمية العنقدة المهجنة Hybrid Clustering Algorithm HCA}

لتحسين استخدام خوارزمية kmeans في كثف التطفل تم دمجها مع الخوارزمية الجينية حيث تم اعتبار

كل حزمة من حزم بيانات الـKDD99 كروموسوم وكل حزمة تحتوي على 41 صفة:

chromosome $=\{$ duration, protocol type , service , flag, ,...etc $\}$

حيث يتم في البداية تهيئة الجيل البدائي ومراكز العناقيد ببيانات عشوائية ثم حساب دالة اللياقة لكل عناصر الجيل

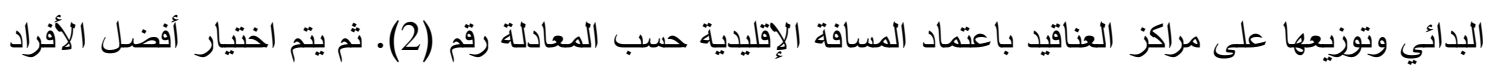

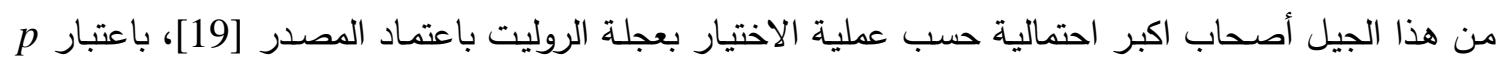
هي احتمالية النجاح و p-1 هي احتمالية الفثل فأن: $F(x)= \begin{cases}0, & \text { if } x<0, \\ 1-p, & \text { if } 0 \leq x<1, \\ 1, & \text { if } 1 \leq x\end{cases}$

ويتم حساب p لكل كروموسوم عن طريق قسمة قيمة دالة اللياقة الخاصـة بهذا الكروموسوم على المجموع الكلي لقيم دالة اللياقة الخاصة بالجيل. ثم يتم تطبيق عمليات التزاوج والطفرة الوراثية على الكروموسومات حسب احتمالية التية معينة لكي يتم تكوين الجيل التالي حيث يتم اخذ أفضل الأفراد من الجيل السابق وإهمال الأسوأ وأيضا يتم تعديل

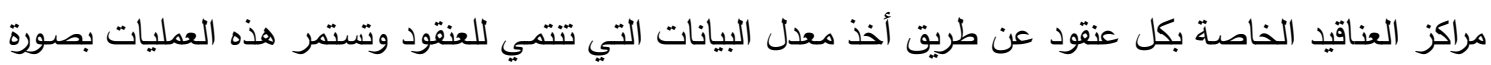

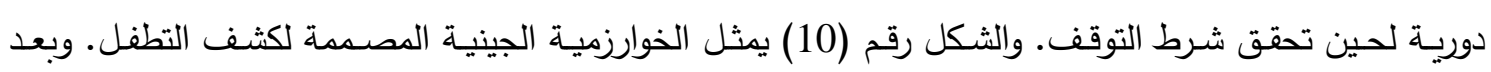
الحصول على أفضل العناقيد يتم تصنيف البيانات على أساسها وذلك بأخذ المسافة الإقليدية بين العناقيد الناتجة

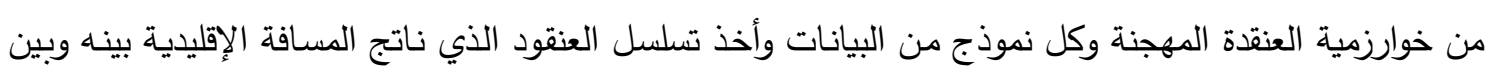

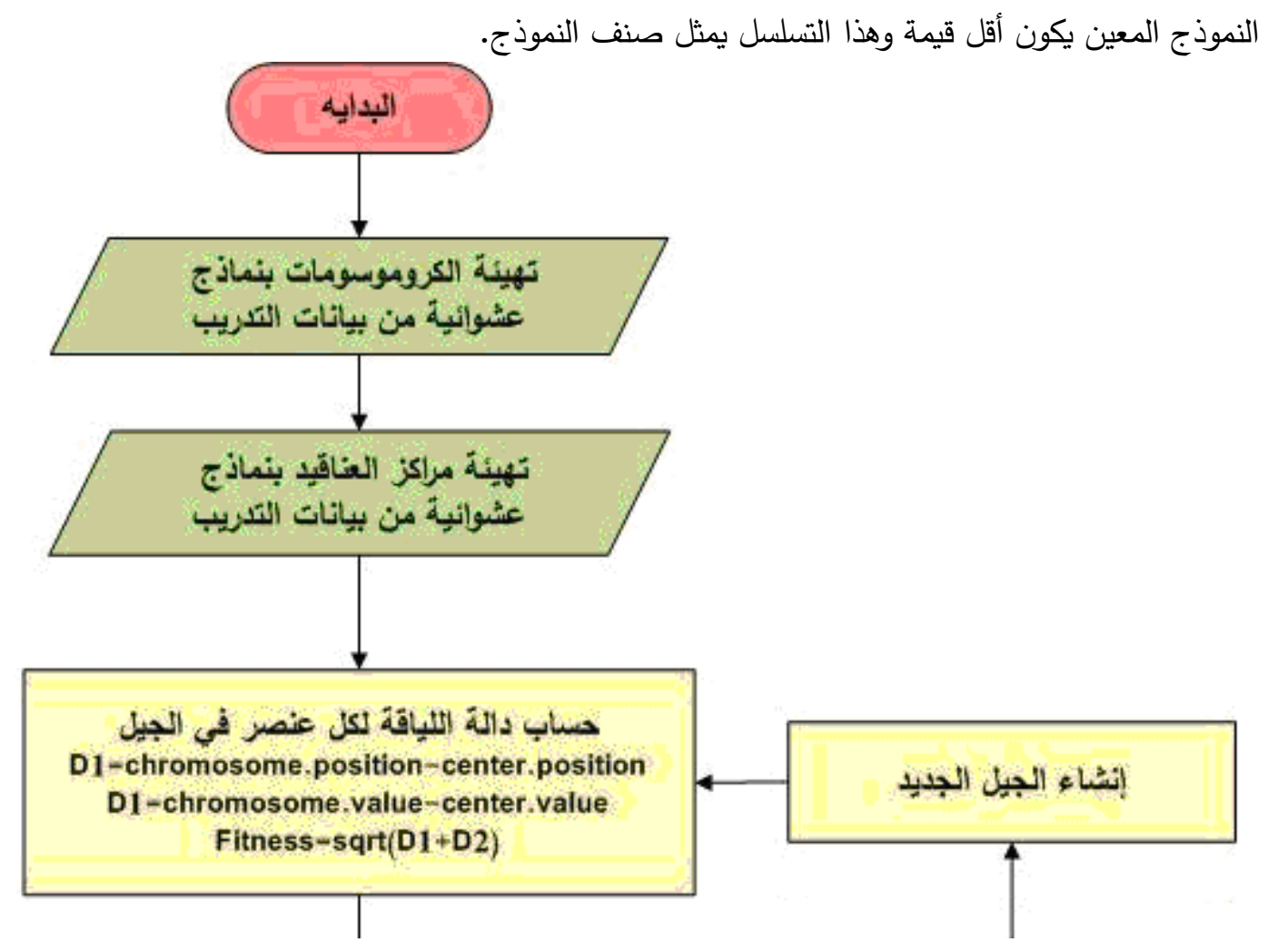




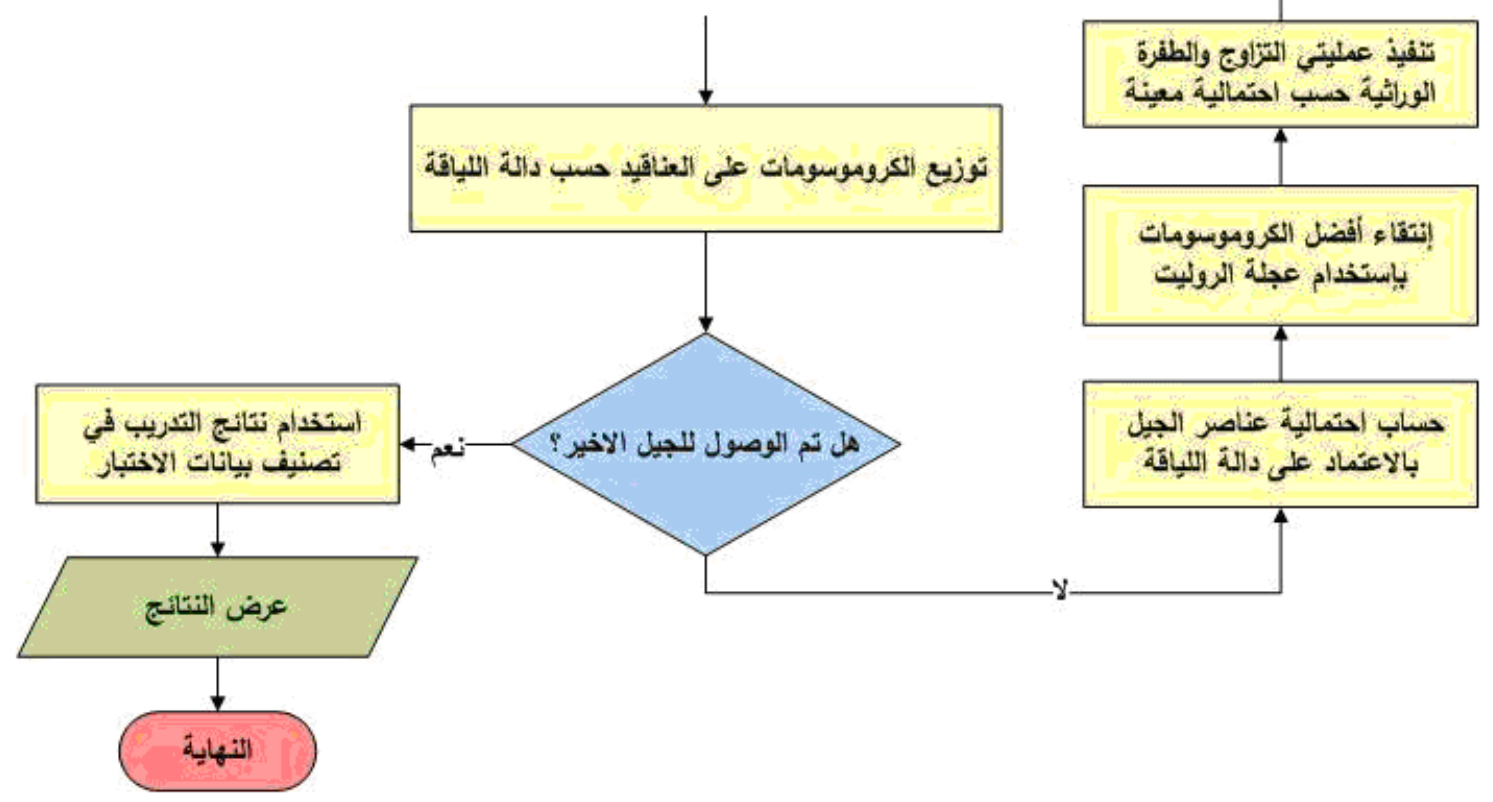

الثكل رقم (10). يمثل الهخط الانسيابي للخوارزمية المستخدمة في نظام كثف التطفل.

\section{3 خوارزمية سرب الطيور Particle Swarm Optimization}

أساس عمل هذه الخوارزمية الأفراد Population حيث تقوم بمحاكاة السلوك الطبيعي لأسراب الطيور في برنامج حاسوبي. يتم في البداية تهيئة الأفراد بحلول عشوائية، تسمى جسيمات Particles كل جسيم من هذهِ

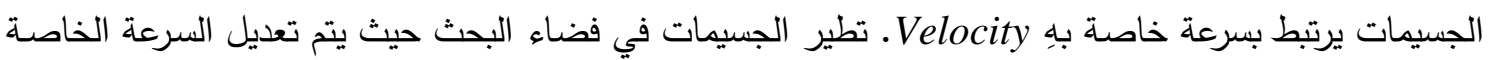

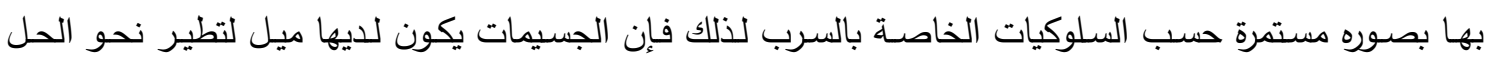
الأفضل في فضاء البحث. كل جسيم في السرب له الخصائص التالية: x ا بمثل الموقع الحالي للجسيم. •vi

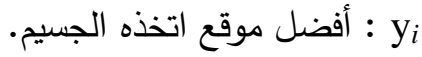

لنفرض أن f هي دالة اللياقة و t هو الزمن الحالي فإن أفضل موقع اتخذه الجسيم يتم تحديثه كالأتي: $y_{i}(t+1)= \begin{cases}y_{i}(t) & \text { if } f\left(x_{i}(t+1) \geq f\left(y_{i}(t)\right)\right. \\ x_{i}(t+1) & \text { if } f\left(x_{i}(t+1)\right)<f\left(y_{i}(t)\right)\end{cases}$

ومن ثم أيجاد أفضل موقع أتخذه الجسيم بالنسبة للسرب بأكمله بواسطة المتجه $\hat{y}(t) \in\left\{y_{0}, y_{1}, \ldots, y_{s}\right\}=\min \left\{f\left(y_{0}(t)\right), f\left(y_{1}(t)\right), \ldots, f\left(y_{s}(t)\right)\right\}$

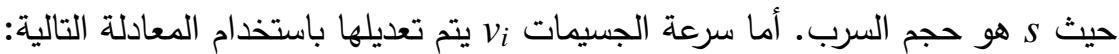
$v_{i, j}(t+1)=w v_{i, j}(t)+c_{1} r_{1, j}(t)\left(y_{i, j}(t)-x_{i, j}(t)\right)+c_{2} r_{2, j}(t)\left(\hat{y}(t)-x_{i, j}(t)\right)$

حيث $i$ تمثل الجسيم و c2 $c_{2}$ c $c_{1}, c_{2}$ و 
يكون المسؤول عن التحكم في زيادة ونقصان البحث الثامل. و تحديث موقع الجسيم i وهو xi بالمعادلة التالية:

$$
x_{i}(t+1)=x_{i}(t)+v_{i}(t+1)
$$

يمكن استخدام مقاييس كثيرة لقياس جودة خوارزميات العنقدة, مقياس الأداء الأكثر شيوعا هو مقياس الخطأ الكمي

$J e=\frac{\sum_{k=1}^{K} \sum \forall z p \in C_{k} d\left(z_{p}, m_{k}\right) / n k}{K}$

حيث $C_{k}$ تمثل العنقود k و $n_{\text { }}$ هو عدد حزم البيانات التي تتتمي للعنقود k و Zp تمثل حزم البيانات. في

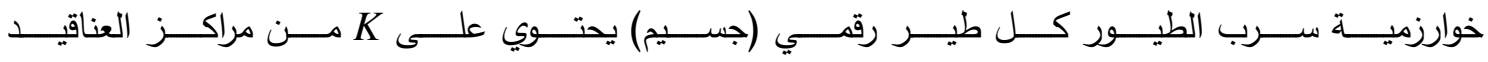

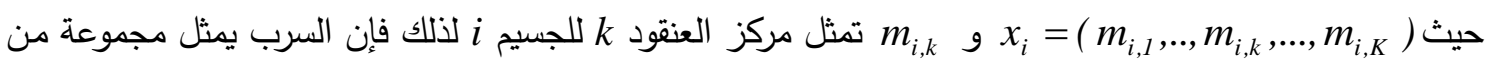
الحلول المرشحة لعملية العنقدة. دالة اللياقة لكل جسيم يمكن حسابها بالمعادلة التالية : $f\left(x_{i}, Z_{i}\right)=w_{1} \bar{d}_{\text {max }}\left(Z_{i}, x_{i}\right)+w_{2}\left(z_{\max }-d_{\text {min }}\left(x_{i}\right)\right)+w_{3} J_{e}$

حيث أن zax $\bar{d}_{\max }\left(Z_{i}, x_{i}\right)=\max _{k=1, \ldots K}\left\{\sum_{\forall z_{p} \in C_{i, k}} d\left(z_{p}, m_{i, k}\right) / n_{i, k}\right\}$ $w_{1}, w_{2}, w_{3}$

وهي أعلى قيمة لمعدل المسافة الإقليدية لمراكز العناقيد في الجسيمات والبيانات المرتبطة بها حيث أن الن

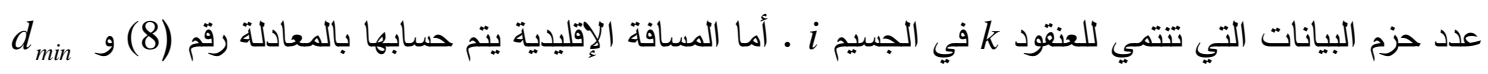
$d_{\min }\left(x_{i}\right)=\min _{\forall k, k k, k \neq k k}\left\{d\left(m_{i, k}, m_{i, k k}\right)\right\}$

دالة اللياقة في المعادلة رقم (10) تقوم بتقليل الخطأ الكمي Je عن طريق تصغير المسافة بين مراكز العناقيد والبيانات التي تتتمي إليها

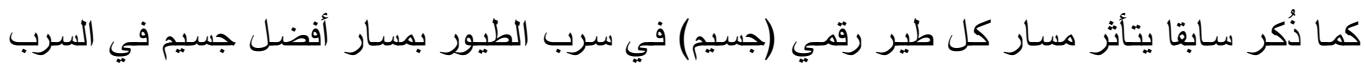
بأكمله إذ تتجذب كل جسيمات السرب بسرعة وفي وقت واحد لأفضل جسيم في فضاء البحث ولكن هناك مشكلة

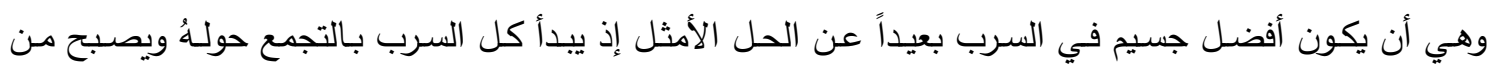

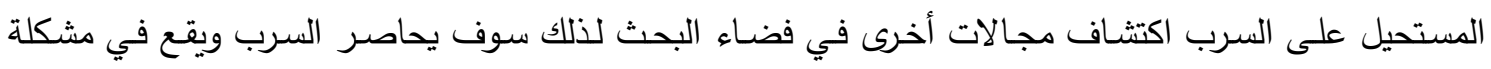

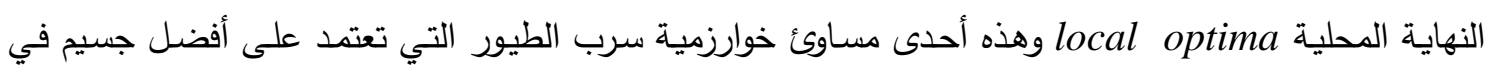
السرب بأكمله global best PSO • يوجد أسلوب آخر لخوارزمية سرب الطيور وهي أن يتأثر مسار كل طير

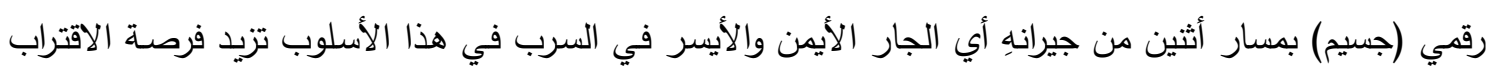

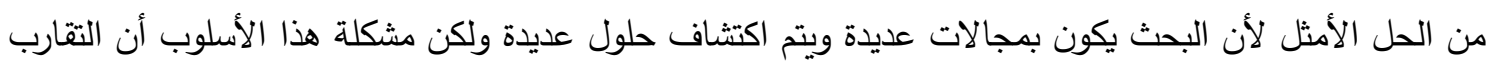
يكون بطيئًاً جداً وتسمى local best PSO [17]. الثكل رقم (11) يمثل المخطط الانسيابي لخوارزمية سرب 


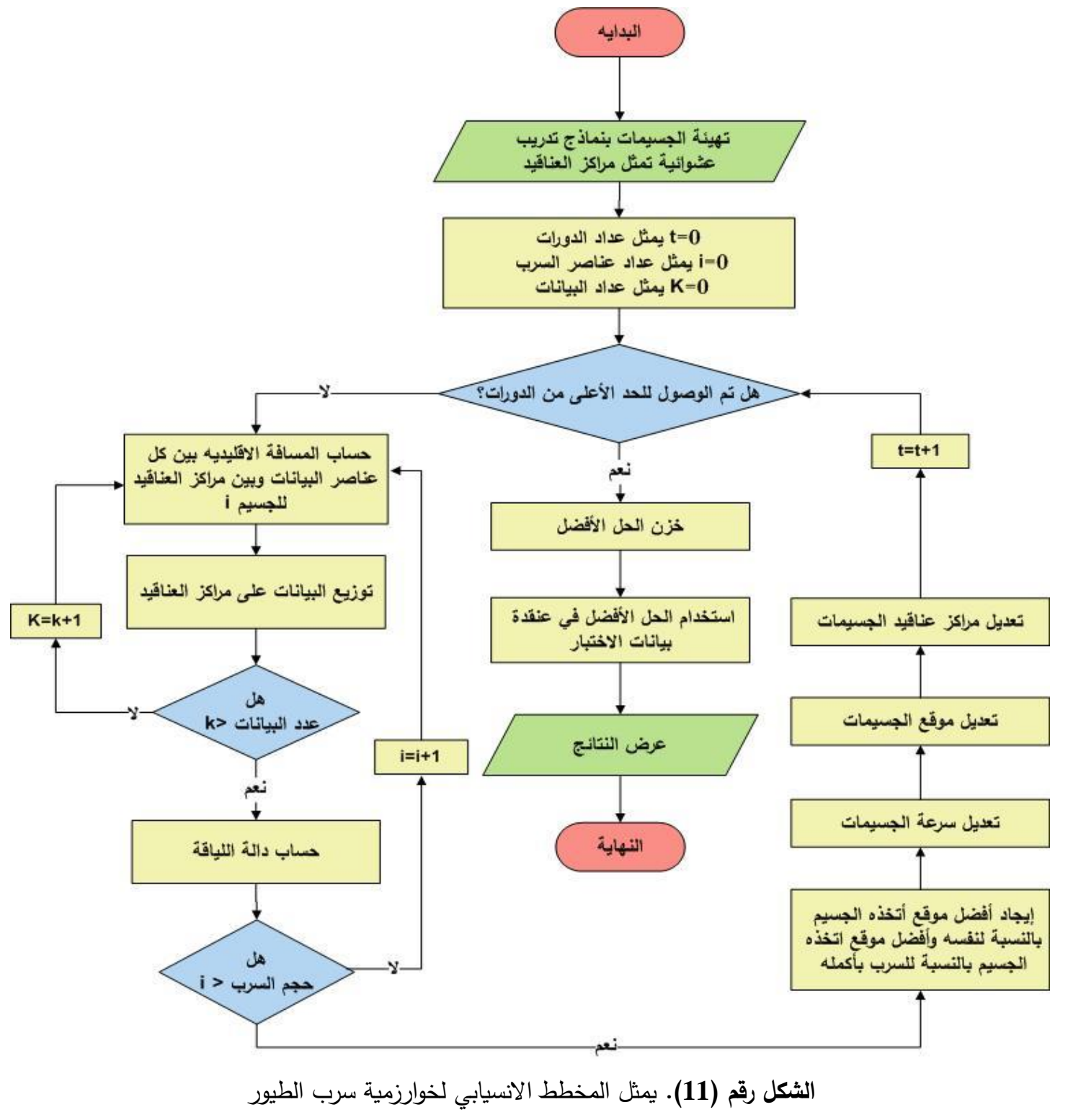

5.3 خوارزمية سرب الطيور المطورة MPSO

بعد دراسة خوارزمية السرب بأنواعها الـ local best PSO و الـglobal best PSO تم التوصل إلى

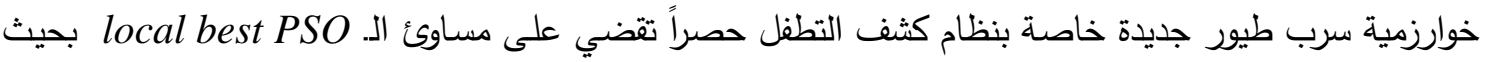

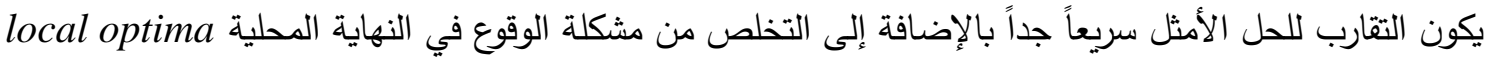
التي تعاني منها الـو global best PSO • إذ تم استبدال دالة اللياقة في الخوارزمية الأصلية المعادلة (10) بمقياس كثف التطفل detection rate الذي يتم قياسهُ بالمعادلة رقم (1) في كل دورة من دورات الخوارزمية يتت البحث عن الحل الأفضل في السرب إذ أن الطير الرقمي الذي يمتلك نسبة كثف تطفل أعلى من البقية يعد

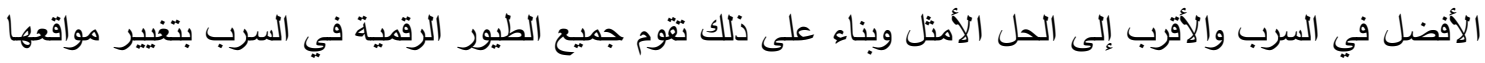
وسرعاتها حسب المعادلات رقم (7,8) نسبة إلى الطير الأفضل لكي يقترب جميع السرب من الحل الأفضل. ففي لوني بداية دورات مرحلة التدريب لوحظ تباين كبير بنسب كثف التطفل لدى الطيور الرقمية وبعد الوصول للدورة التاسعة

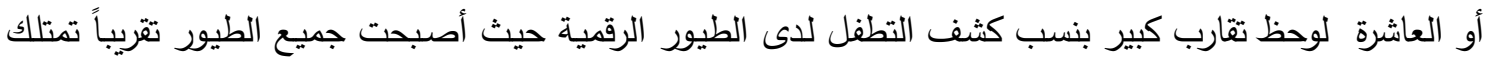


نسبة كثف تطفل 99\% و 100\% وفي النهاية تم اختيار أفضل طير رقمي صاحب نسبة الـ 100\% الذي يعد هو الحل الأفضل لمشكلة كثف التطفل.الثكل رقم (12) يمثل المخطط الانسيابي لخوارزمية سرب الطيور المطورة الخاصة بنظام كثف التطفل.

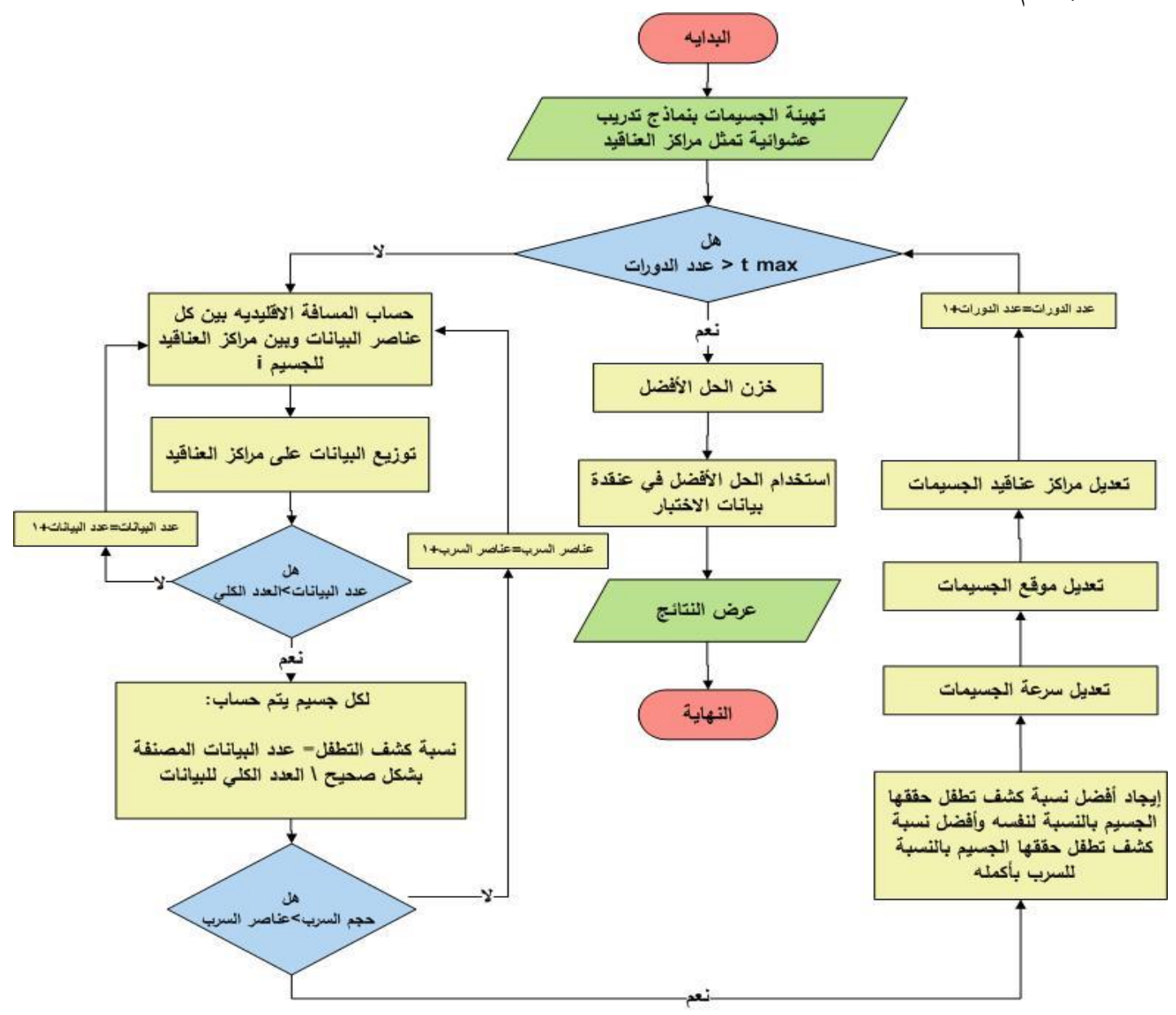

الثكل رقم (12). يمثل المخطط الانسيابي لخوارزمية سرب الطيور المطورة

4. التنفيذ والاختبار Implementation and Testing

تم تتفيذ النظام على 9000 عينة من بيانات KDD99 حيث تم اختيار 5000 منها للتدريب و 4000 للاختبار كما في الجدول الآتي:

\begin{tabular}{|c|c|}
\hline عددها في الملف| & نسبتها في الملف 1500 \\
\hline$\% 37,5$ & 2500 \\
\hline$\% 62,5$ & 4000 \\
\hline$\% 100$ & \\
\hline
\end{tabular}

\begin{tabular}{|c|c|c|}
\hline نسبتها في الملف & عددها في الملف & نوع الحزمة \\
\hline$\% 40$ & 2000 & normal \\
\hline$\% 60$ & 3000 & abnormal \\
\hline$\% 100$ & 5000 & العدد الكلي \\
\hline
\end{tabular}

أ)جدول محتويات ملف التدريب لكثف الهجمات ب) جدول محتويات ملف الاختبار لكثف الهجمات الجدول رقم (1). يمثل محتويات ملفات كثف الهجمات المستخدمة في النظام 
تم تدريب خوارزمية العنقدة kmeans وكذلك خوارزمية العنقدة المهنـة بالخوارزمية الجينية على5000

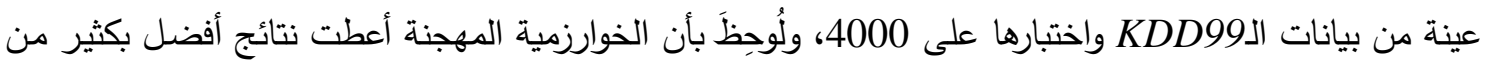
خوارزمية العنقدة kmeans حيث تم الحصول على النتائج التالية:

(مرحلة الاختبار )

\begin{tabular}{|c|c|}
\hline HCA & KM \\
\hline$\% 90,6$ & $\% 73,72$ \\
\hline 0 & 51 \\
\hline 624 & 0 \\
\hline 1876 & 2551 \\
\hline 2124 & 1449 \\
\hline$\% 24,96$ & $\% 0$ \\
\hline$\% 0$ & $\% 3,4$ \\
\hline$\% 100$ & $\% 96,6$ \\
\hline$\% 75,04$ & $\% 100$ \\
\hline 1 & 0,9804 \\
\hline 0,8651 & 0,9874 \\
\hline
\end{tabular}

(مرحلة التدريب) (مبان)

\begin{tabular}{|c|c|c|}
\hline HCA & KM & \% الS \\
\hline$\% 97,52$ & $\% 78.98$ & DR \\
\hline 0 & 51 & FP \\
\hline 124 & 0 & FN \\
\hline 2876 & 3051 & TP \\
\hline 2124 & 1949 & TN \\
\hline$\% 4,13$ & $\% 0$ & FNR \\
\hline$\% 0$ & $\% 2,55$ & FPR \\
\hline$\% 100$ & $\% 97,45$ & TNR \\
\hline$\% 95,87$ & $\% 100$ & TPR \\
\hline 1 & 0.9836 & Precision \\
\hline 0.9758 & 0.9899 & Accuracy \\
\hline
\end{tabular}

الجدول رقم (2). يمثل نتائج عملية تدريب واختبار خوارزميتي العنقدة والعنقدة المهجنة بالخوارزمية الجينية

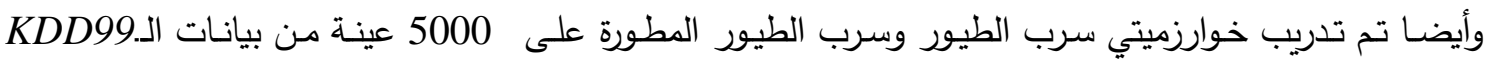

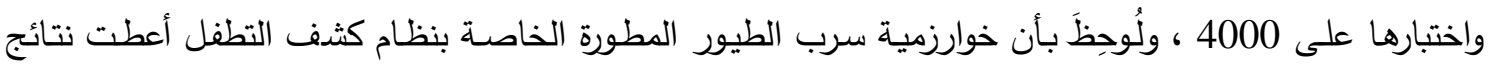

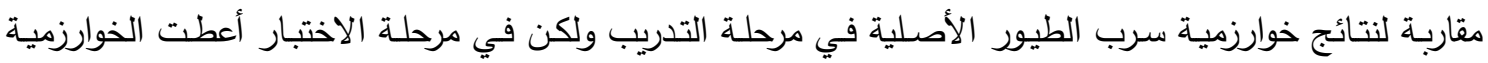
المطورة نتائج أفضل بكثير من الخوارزمية الأصلية إذ تم الحصول على النتائج التالية:

(مرحلة الاختبار)

\begin{tabular}{|c|c|}
\hline MPSO & PSO \\
\hline$\% 100$ & $\% 96,37$ \\
\hline 0 & 145 \\
\hline 0 & 0 \\
\hline 1500 & 2645 \\
\hline 2500 & 1355 \\
\hline$\% 0$ & $\% 0$ \\
\hline$\% 0$ & $\% 9,67$ \\
\hline$\% 100$ & $\% 90,33$ \\
\hline$\% 100$ & $\% 100$ \\
\hline 1 & 0,948 \\
\hline 1 & 0,965 \\
\hline
\end{tabular}

الجدول رقم (3). يمثل نتائج عملية تدريب واختبار خوارزميتي سرب الطيور وسرب الطيور المطورة
(مرحلة التدربب)

\begin{tabular}{|c|c|c|}
\hline MPSO & PSO & \% الs \\
\hline$\% 100$ & $\% 96,96$ & DR \\
\hline 0 & 152 & FP \\
\hline 0 & 0 & FN \\
\hline 3000 & 3152 & TP \\
\hline 2000 & 1848 & TN \\
\hline$\% 0$ & $\% 0$ & FNR \\
\hline$\% 0$ & $\% 7,6$ & FPR \\
\hline$\% 100$ & $\% 92,4$ & TNR \\
\hline$\% 100$ & $\% 100$ & TPR \\
\hline 1 & 0,954 & Precision \\
\hline 1 & 0,9705 & Accuracy \\
\hline
\end{tabular}


من النتائج أعلاه نستتتج أن أفضل خوارزمية من الخوارزميات الأربعة التي تم عرض نتائجها هي خوارزمية سرب

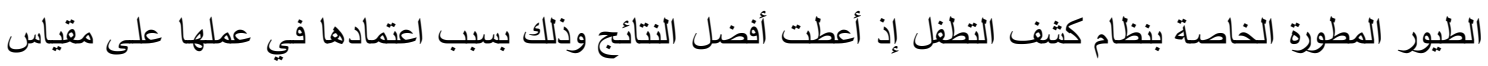
كثف التطفل.

\begin{tabular}{|c|c|c|}
\hline Execution time & DR & الطريقة \\
\hline 3.4 seconds & $\% 73,72$ & KM \\
\hline 20.5 seconds & $\% 90,6$ & HCA \\
\hline 1975.5 seconds & $\% 96,37$ & PSO \\
\hline 1336.9 seconds & $\% 100$ & MPSO \\
\hline
\end{tabular}

الجدول رقم (4). يمثل مقارنة بين الطرق الأربعة من ناحية نسبة كثف التطفل في مرحلة الاختبار والوقت المستغرق في عملية التدريب ناحبة نسبة كئب

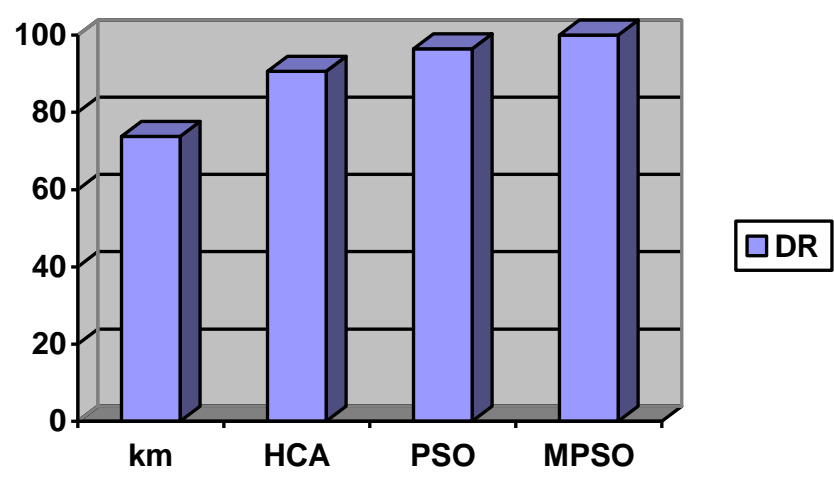

الثكل رقم (13). يمثل مخطط المقارنة للطرق الأربعة حسب مقياس كثف التطفل

5. الاستنتاجات

بعد تطبيق طرائق كثف التطفل التي تعتمد على التقنيات الذكائية باستخدام خوارزمية سرب الطيور

والخوارزمية الجينية على مجموعة بيانات KDDcup99 لوجِظ ما يلي:

• بالنسبة لطريقة العنقدة التقليدية المتمثلة باستخدام خوارزمية Kmeans أعطت نتائج مقبولة إلى حد ما ولكنها

ليست بالمستوى المطلوب, وذلك اتضح من خلال حساب قيم مقاييس أداء النظام مثل نسبة الكثف ونسبة

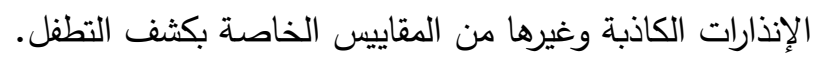

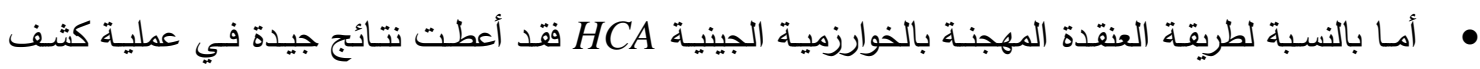

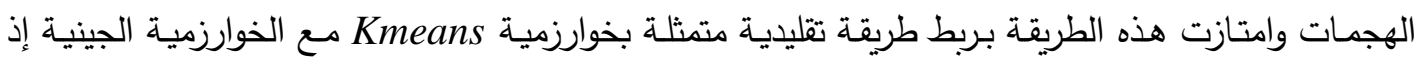

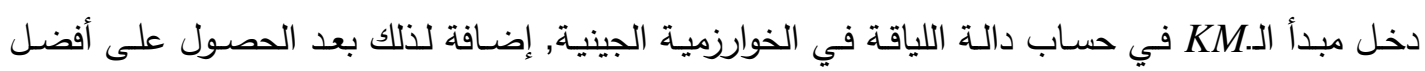
العناقيد في الخوارزمية المهنة HCA يتم تمرير جميع البيانات على هذه العناقيد وحساب المسافة الإقليدية

$$
\text { ومن ثم كثف الهجمات. }
$$

تم استخدام خوارزميـة سرب الطيور لأجراء عمليـة كثف الهجمـات وذلك بتتفيذ الخوارزميـة على مجموعـة بيانات والحصول على الحل الأمثل, وهذه الطريقة أعطت نتائج أفضل من الطريقتين أعلاه, ولكن هناك مشكلة

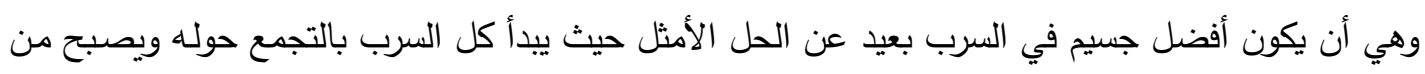


المستحيل على السرب اكتثاف مجالات أخرى في فضاء البحث لذلك سوف يحاصر السرب ويقع في مشكلة النهاية المحلية local optima.

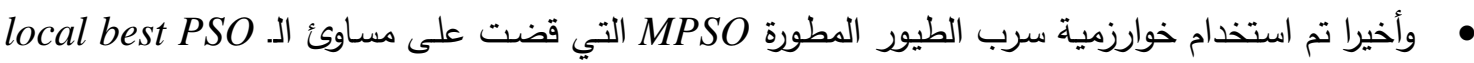
بحيث يكون التقارب للحل الأمثل سريع جداً بالإضـافة إلى التخلص من مشكلة الوقوع في النهايـة المحلية local optima الطيور بمقياس كثف التطفل detection rate الذي إذ المقياس الأهم في أنظمة كثف التطفل. وأعطت هذه الطريقة أفضل النتائج بجميع المقاييس المستخدمة حيث وصلت نسبة الكثف إلى 100\% ولم تؤدِد للوقوع في مشكلة النهاية المحلية. 


\section{|"مصادر}

[1] Wei Li " Using Genetic Algorithm for Network Intrusion Detection" Department of Computer Science and Engineering Mississippi State University, Mississippi State, MS 39762 Email: wli@ cse.msstate.edu.

[2] Rozenblum D. , 2001 , "understanding Intrusion Detection System", SANS Institute.

[3] Anderson R. J. , 2008 , " Security Engineering: A Guide to Building Dependable Distributed Systems", Second Edition.

[4] Dr. Herrero A. and Prof.Dr. Corchado E. , 2011 "Mobile Hybrid Intrusion Detection" Springer.

[5] Topark M. ,2009, "Intrusion Detection System Alert Correlation with Operating System Level Logs" A Thesis Submitted to The Graduate School of Engineering and Sciences of İzmir Institute of Technology .

[6] http://www.windowsecurity.com

[7] Cramer L.M , Cannady J. and Harrell J. , 1996, "New Methods of Intrusion Detection using Control- Loop Measurement" , Georgia Institute of Technology Atlanta .

[8] Wilson J., Dudley P. and Khan B. , 2008, "Requirements Specification " , CQFQMT .

[9] Odutola K. , Oguntimehin A. , Tolke L. and Wulp M. V. , 2008 , "ArgoUML Quick Guide " .

[10] Kang D. , Fuller D. and Honavar V. ,2005, "Learning Classifiers for Misuse and Anomaly Detection Using a Bag of System Calls Representation" , IEEE .

[11] Norsyafawati F. , Norwawi N. and Seman K. , 2011, "Identifying False Alarm Rates for Intrusion Detection System with Data Mining" , IJCSNS International Journal of Computer Science and Network Security, VOL.11 No.4.

[12] Hammersland R. , 2007, "ROC in Assessing IDS Quality" , Norwegian Information Security Lab, Gjøvik University College.

[13] Chang R, Lai L. , Wang J. and Kouh J. , 2007, "Intrusion Detection by Backpropagation Neural Networks with Sample-Query and Attribute-Query", International Journal of Computational Intelligence Research. Vol.3, No. 1 .

[14] Adetunmbi A., Adeola S. and Daramola O. ,2010 ,"Analysis of KDD '99 Intrusion Detection Dataset for Selection of Relevance Features" , Proceedings of the World Congress on Engineering and Computer Science Vol I .

[15] Vescan A. and HORIA F. , 2008, "Constraint Optimization-Based Component Selection problem" , INFORMATICA,Volume LIII, No. 2.

[16] Izenman A. J. ,2008,"Modern Multivariate Statistical Techniques", Springer .

[17] Abraham A. , Grosan C and Ramos V. ,2006, "Swarm Intelligence in Data Mining", Springer.

[18] Vinu V. ,Thomas G. and Lumban F. , 2011,"Information Technology and Mobile Communication" Springer, International Conference.

[19] Shonkwiler R. W. and Mendivil F. ,2009, "Explorations in Monte Carlo Methods",Springer . 